\title{
Wavelets-based damage localization on beams under the influence of moving loads
}

\author{
José Manuel Machorro-López, Andrea Bellino ${ }^{a}$, Stefano Marchesiello \\ AND Luigi GaRiBALDi
}

Department of Mechanical and Aerospace Engineering, Politecnico di Torino, Italy

Received 27 November 2012, Accepted 11 December 2012

\begin{abstract}
Early damage detection on structures plays a very important role for ensuring safety and reliability. This paper provides an efficient method based on wavelet transforms in order to detect and localize damage on structures subjected to moving loads such as beams and bridges. A numerical model based on the experimental test-rig utilized in this study is developed by using a finite element commercial software. Different types of damage on the bridge of the numerical model are simulated and transient analyses are performed by incorporating a load which moves constantly along the beam nodes. Continuous wavelet transform diagrams using the vertical acceleration responses show that damage can be identified and localized even with significant percentages of noise. Nevertheless, the method is improved by filtering the signals, removing the border effects, and calculating the total wavelet energy of the beam from the coefficients along the selected range of scales. Thus, the accumulation of wavelet energy could indicate the presence of damage. Finally, laboratory experiments are conducted to validate this work and a good agreement between numerical and experimental results is obtained.
\end{abstract}

Key words: Damage / beams / moving loads / wavelets / finite element

\section{Nomenclature}

\begin{tabular}{|ll|}
\hline$a:$ & scale parameter of the wavelets \\
$b:$ & translation parameter of the wavelets \\
$b_{\mathrm{b}}:$ & base of the transverse section of the beam $(\mathrm{m})$ \\
$c_{j}:$ & coefficient of the wavelets \\
$f_{\mathrm{s}}:$ & sampling frequency $(\mathrm{Hz})$ \\
$h_{\mathrm{b}}:$ & height of the transverse section of the beam $(\mathrm{m})$ \\
$L_{\mathrm{b}}:$ & total length of the beam $(\mathrm{m})$ \\
$P:$ & weight of the moving load $(\mathrm{N})$ \\
$p:$ & relative crack depth \\
$t:$ & time $(\mathrm{s})$ \\
$v:$ & linear speed $\left(\mathrm{m}^{-1}\right)$ \\
$x:$ & horizontal position of the moving load $(\mathrm{m})$ \\
\hline
\end{tabular}

\section{Introduction}

All the load-carrying structures accumulate damage that is usually caused by deterioration of some components during their service life. The particular case of the bridges has been intensively studied among many others

\footnotetext{
a Corresponding author: andrea.bellino@polito.it
}

since a damage that is not early detected in this kind of civil constructions may lead to structural failure and the loss of human lifes besides the huge economic losses. Many accidents with catastrophic consequences due to collapse of bridges have occurred even in the most developed countries. Recently, the eight-lane steel truss arch bridge known as Bridge 9340 failed during the evening rush hour on August 1, 2007, collapsing to the Mississippi River in Minneapolis, Minnesota, USA. Thirteen people were killed and 145 were injured [1].

Therefore, there is a need of providing an efficient method to assess the structural state of bridges. The implementation of damage detection strategies based on analyses of the vibration measurements for structural health monitoring (SHM) have been widely used in the last three decades. Several researchers like Farrar and Jauregui [2] have presented comparative studies of damage identification algorithms applied to a bridge, and found that standard modal properties such as resonance frequencies and mode shapes are poor damage indicators.

Liew and Wang [3] notified that crack identification on simply supported beams is more efficient by using wavelet transforms (WT) instead of eigenvalues analysis. Zhu and Law [4] proposed a method for crack identification on bridges under a moving load using wavelet analysis. The 
locations of the cracks were determined from the sudden changes in the spatial variation of the transform responses; whereas their sizes were estimated from the magnitude of the coefficients of the WT diagrams. On the other hand, Surace and Ruotolo [5] developed a technique using the WT to detect a single crack in a simple cantilever beam. Likewise, Quek et al. [6] presented WT-based analyses to accurately identify small cracks in beams under both simply supported and fixed-fixed boundary conditions. Douka et al. [7] used a WT-based method to determine the location and size of a crack in a beam using the fundamental mode of vibration. Similarly, Gentile and Messina [8] showed that by means of the WT it is possible to detect and localize damage as well as estimate its size from both noisy and clean data.

This article presents a new approach based on WT in order to provide an efficient method to identify and localize damage on structures subjected to moving loads. A numerical model considering the experimental test-rig utilized in this study is developed by using the software ANSYS, which is based on the finite element method (FEM). Transient analyses for healthy and damaged cases are performed by incorporating a load which moves along the beam nodes at constant speed. The acceleration responses are collected for the complete duration of the load crossing the beam.

A MATLAB code is written to post-process the data obtained from the simulations. Continuous wavelet transform (CWT) diagrams show that if a convenient mother wavelet is selected, damage can be detected and localized. The method is improved by filtering the noisy signals, removing the border effects from the CWT diagrams, and selecting an adequate range of scales. In this way, the wavelet diagrams become clearer to identify damage and the total wavelet energy of the beam is obtained from the coefficients along the selected range of scales for each point of the beam. The results indicate that the total wavelet energy for an undamaged beam is very flat; in contrast, prominent peaks of wavelet energy in the neighborhood of the damage are observed for damaged beams. Lastly, an average of the total wavelet energy is calculated for a few different points of measurement and it is shown that the percentage of error between the position of the maximum energy for damaged cases and the damage location is very small.

Finally, experiments are conducted to validate this work using a test-rig composed by an aluminum bridge whose supports try to simulate a simply supported beam. The vertical responses are acquired while a plastic wagon passes over the bridge. The damage detection method used for the FEM simulations is applied also for the experiments and a good agreement between both results is obtained.

\section{Fundamentals of wavelet transform}

The fast Fourier transform (FFT) is a useful tool to analyze the frequency components of a signal. However, since the FFT is the sum over all time of a signal $f(t)$ multiplied by a complex exponential (see (1)), the main disadvantage of using this transform is that we cannot know the instant that a particular frequency rises.

$$
F(w)=\int_{-\infty}^{\infty} f(t) \mathrm{e}^{-j w t} \mathrm{~d} t
$$

The Fourier transform can provide accurate frequency information, but no time information. On the other hand, good time estimation can be accomplished by applying the short time Fourier transform (STFT); however, the width of window is fixed and limits the resolution in frequency.

The wavelet transform (WT) is a powerful tool in the field of signal processing for SHM which overcomes the restriction of the fixed time-frequency resolution. This is because WT are based on a windowing technique with variable-sized regions, which is capable of using long time intervals where precise low frequency information is needed, and short time intervals where precise high frequency information is desired. The WT are based on small wavelets with limited duration known as mother wavelets, they are the basis functions in wavelet analysis and are defined by two parameters: translation and scale [9]. For a wavelet of order $N$, the mother wavelet can be represented as:

$$
\psi(n)=\sum_{j=0}^{N-1}(-1)^{j} c_{j}(2 n+j-N+1)
$$

where $c_{j}$ represents the coefficient. Thus, the mother wavelet has to satisfy two conditions: it must integrate to zero, that is:

$$
\int_{-\infty}^{\infty} \psi(t) \mathrm{d} t=0
$$

and it must be square integrable or, equivalently, it must have finite energy, that is:

$$
\int_{-\infty}^{\infty}|\psi(t)|^{2} \mathrm{~d} t<\infty
$$

On the other hand, the continuous wavelet transform (CWT) is defined as:

$$
W(a, b)=\frac{1}{\sqrt{a}} \int f(t) \psi^{*}\left(\frac{t-b}{a}\right) \mathrm{d} t
$$

where $a$ and $b$ are the scale and translation parameters, respectively; whereas $\psi^{*}$ is the complex conjugate of $\psi$. Then, the mother wavelet $\psi$ can be represented as:

$$
\psi_{j, k}(t)=2^{j / 2} \psi\left(2^{j} t-k\right)
$$

Likewise, in order to obtain an inverse wavelet transform, the mother wavelet has to satisfy the admissibility condition, which is defined as:

$$
\int_{-\infty}^{\infty} \frac{|\Psi(w)|^{2}}{|w|} \mathrm{d} w<\infty
$$




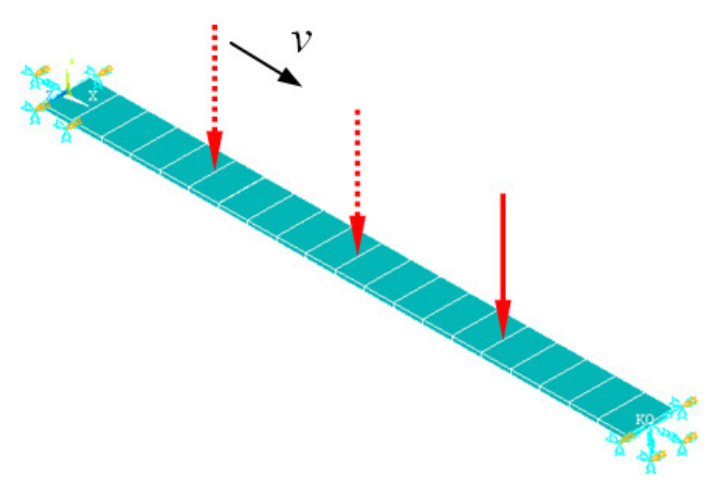

Fig. 1. Final FEM model with constant load moving along the beam nodes.

where $\Psi$ is the Fourier transform of $\psi$, then (5) can be represented as:

$$
W(a, b)=\left\langle f(t), \psi_{a, b}^{*}(t)\right\rangle
$$

Thus, the CWT is a collection of inner products of a signal $f(t)$ and the translated and dilated wavelets $\psi_{a, b}(t)$. The value of the scale $a$ is proportional to the reciprocal of the frequency, which results from (9); where $F$ [ ] denotes the Fourier transform:

$$
F[\psi(t / a)]=|a| \psi(a w)
$$

\section{FEM-based model and simulations}

A versatile model was created with the FEM-based software ANSYS to represent the experimental test-rig used for this study. The $1.86 \mathrm{~m}$ length bridge was simulated by using 2-D elastic beam elements type BEAM3, whereas the supports were represented by incorporating COMBIN14 spring-damper elements at the ends of the beam. The damping of the supports was neglected and the values of stiffness were adjusted according with the configuration of the test-rig and its equivalent natural frequencies.

Damage on the bridge was simulated by changing the cross-sectional geometric characteristics of one element equivalent to $5 \%$ of the beam length. Furthermore, the wagon which passes over the experimental bridge was characterized as a load that moves at constant speed along the beam nodes. A code was written in ANSYS which allows easily changing the parameters of the model and performing different types of solutions. The complete FEM model can be seen in Figure 1, which contains 28 elements.

The acceleration vertical responses from the transient analysis were collected in the time domain for all the beam nodes and for the complete lasting of the load crossing the beam. The transient simulations were performed by establishing the weight of the moving load $P=24.1 \mathrm{~N}$ and a sampling frequency $f_{\mathrm{s}}$ of $300 \mathrm{~Hz}$, whereas two constant speeds of the load were analyzed: $v=0.2 \mathrm{~m} . \mathrm{s}^{-1}$ and $0.5 \mathrm{~m} . \mathrm{s}^{-1}$. All the scenarios simulated in this section
Table 1. Scenarios numerically simulated with ANSYS.

\begin{tabular}{ll}
\hline Case & \multicolumn{1}{c}{ Description } \\
\hline $\mathrm{H}$ & Healthy beam, $v=0.2 \mathrm{~m} \cdot \mathrm{s}^{-1}, P=24.1 \mathrm{~N}$ \\
$\mathrm{D} 1$ & $\begin{array}{l}\text { Cracked beam }(p=0.5 \text { at } 0.66 \mathrm{~m}), v=0.2 \mathrm{~m} . \mathrm{s}^{-1}, \\
P=24.1 \mathrm{~N}\end{array}$ \\
$\mathrm{D} 2$ & $\begin{array}{l}\text { Cracked beam }(p=0.4 \text { at } 0.66 \mathrm{~m}), v=0.2 \mathrm{~m} . \mathrm{s}^{-1}, \\
P=24.1 \mathrm{~N}\end{array}$ \\
D3 & $\begin{array}{l}\text { Cracked beam }(p=0.3 \text { at } 0.66 \mathrm{~m}), v=0.2 \mathrm{~m} . \mathrm{s}^{-1}, \\
P=24.1 \mathrm{~N}\end{array}$ \\
D4 & $\begin{array}{l}\text { Cracked beam }(p=0.2 \text { at } 0.66 \mathrm{~m}), v=0.2 \mathrm{~m} . \mathrm{s}^{-1}, \\
P=24.1 \mathrm{~N}\end{array}$ \\
D5 & $\begin{array}{l}\text { Cracked beam }(p=0.1 \text { at } 0.66 \mathrm{~m}), v=0.2 \mathrm{~m} . \mathrm{s}^{-1}, \\
P=24.1 \mathrm{~N}\end{array}$ \\
D6 & $\begin{array}{l}\text { Cracked beam }(p=0.4 \text { at } 1.40 \mathrm{~m}), v=0.5 \mathrm{~m} . \mathrm{s}^{-1}, \\
P=24.1 \mathrm{~N}\end{array}$ \\
\hline
\end{tabular}

are shown in Table 1 . Then, a MATLAB code was written to post-process the signals obtained from the FEM simulations.

The first set of FEM simulations consisted in comparing a healthy bridge $(\mathrm{H})$ against a damaged bridge with a crack of $50 \%$ of the beam height $(p=0.5)$ located at $0.66 \mathrm{~m}$ from the left end (D1). All the WT analyses performed in this paper were based on using the Mexican hat mother wavelet because it was found to be the most promising one for detecting damage among all the mother wavelets. In Figure 2, the CWT diagrams obtained from the mid-span vertical acceleration responses for the cases $\mathrm{H}$ and D1 with $7 \%$ of white Gaussian noise are shown and it can be observed that there are no evidences of damage in the healthy diagram; whereas for the damaged case, it is clear that its CWT diagram presents an indicator of the damage presence around the damage location. The bright tonalities in the CWT diagrams indicate high magnitudes for the coefficients.

After analyzing the CWT diagrams presented in Figure 2 , the possibilities of detecting and localizing damage with this technique are very heartening, but there are still several aspects that can be improved in order to obtain a more precise and formal method:

1) In order to increase the evidence of damage, a Savitzky-Golay filter is designed and the filtered signals are analyzed instead of the noisy ones.

2) The coefficients of the CWT diagrams are selected as our first damage indicator.

3) The border effects of the CWT diagrams obstruct the damage detection; therefore, this effect is removed by eliminating a small percentage of the first and last part of the filtered signals, then those trimmed signals are padded with the corresponding first and last five cycles, and lastly the padded signals are extended.

4) A convenient range of scales must be selected to analyze the coefficients into a limited area of the CWT diagrams without the influence of the first natural frequency and the higher ones; therefore, a range of scales is chosen from 100 to 200 .

5) The total wavelet energy of the bridge is considered as the principal feature to detect and localize damage. 

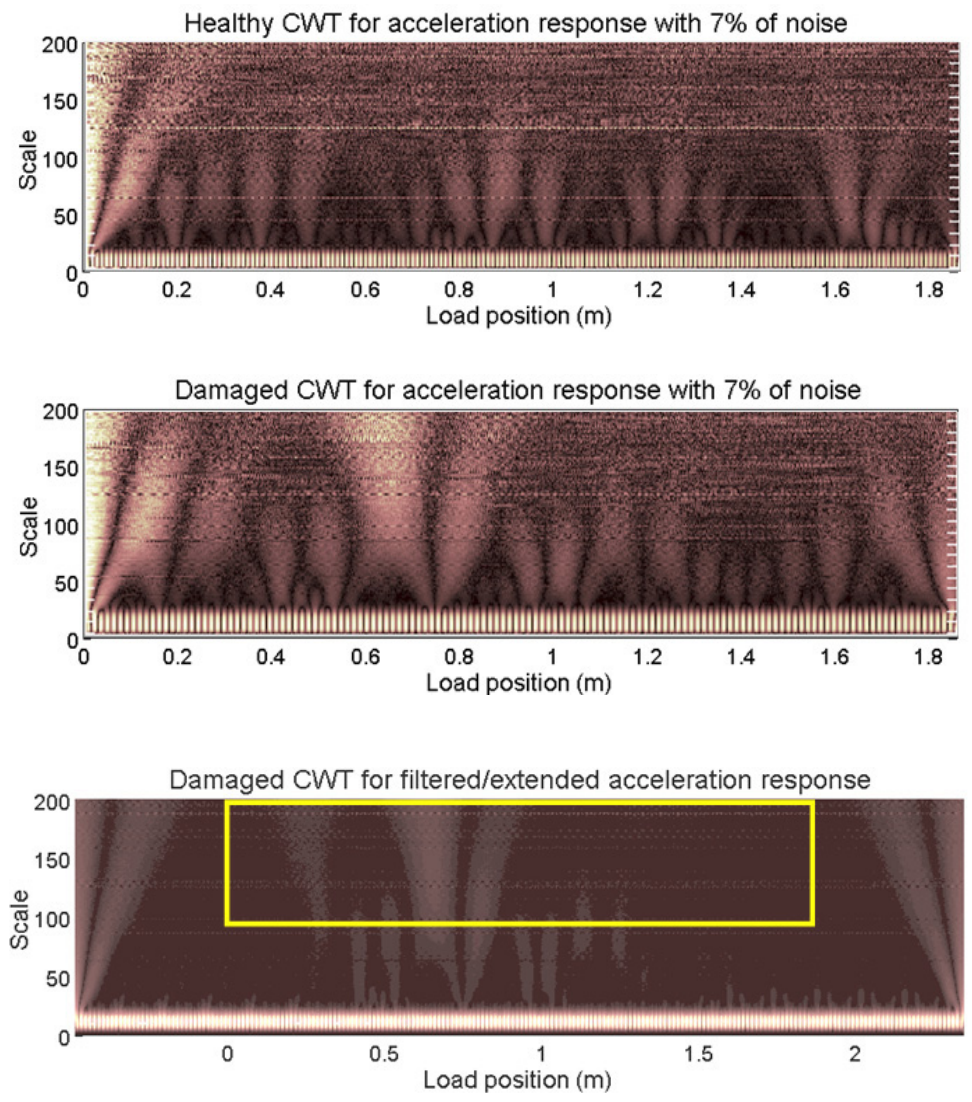

Fig. 2. Mid-span CWT diagrams for healthy case $(\mathrm{H})$ and damaged case (D1) with $7 \%$ of noise.
Fig. 3. Mid-span CWT diagram for damaged case (D1) obtained from acceleration signal after being filtered, trimmed, padded, and extended.
This latter parameter is obtained by calculating the area under the curve formed by the coefficients along the selected range of scales for each point of the beam; thus a significant peak of wavelet energy will indicate the presence of damage. Lastly, an average of the total wavelet energy for different measurement points is obtained to make the analysis and the damage localization more precise.

Figure 3 shows the CWT diagram obtained from the noisy damaged acceleration signal used to generate the damaged CWT diagram shown in Figure 2 but after being filtered, trimmed, padded, and extended. The yellow rectangle attached in Figure 3 indicates the convenient area to calculate the total wavelet energy and detect damage; into this rectangle there are neither the effects of the first natural frequency nor the border effects.

Thus, in Figure 4 it is exhibited the total wavelet energy of both healthy and damaged beams considering 3 points of measurement at $0.25 L_{\mathrm{b}}, 0.5 L_{\mathrm{b}}$, and $0.75 L_{\mathrm{b}}$ (where $L_{\mathrm{b}}$, is the total length of the beam), as well as the corresponding average of the wavelet energy for those points of measurement. The wavelet energies for the healthy case are very flat and there are no parts of the beam with accumulation of energy, and therefore there is no any significant peak of energy which could suggest the presence of damage. On the other hand, for the damaged case, it is evident that for all the points of measurement there exists a significant peak of energy in the neighborhood of the damage; and as a consequence, the maximum level of the average energy occurs at $0.6643 \mathrm{~m}$; therefore, the percentage of error between the position of the maximum peak of the average energy for this damaged case and the location of the damage is $0.65 \%$.

Finally, the average wavelet energies for all the cases presented in Table 1 are shown in Figure 5, where it is possible to see that the maximum peak of energy for all the damaged cases occurs in the vicinity of the corresponding damages locations. The case with the smallest damage (D5) does not present a significant prominent peak of energy but at least its maximum level of energy occurs around the damage location; in fact, following this method, the percentage of error in the localization of damage for the cases D1-D5 was less than $1.40 \%$, whereas the corresponding percentage of error for the case D6 increased until $3.86 \%$ due to the increment of speed and the needing of a better resolution (sampling frequency). Furthermore, the damaged cases D1-D5 have the same conditions and only the intensity of damage was modified; thus, if their energy curves are observed, it is evident that the magnitude of the maximum peak of energy increases if the damage also increases. Therefore, the method here proposed could detect, localize, and classify damage according with its intensity on structures subjected to moving loads.

\section{Experimental validation}

The test-rig [10] utilized to validate this work consisted in two rigid bridges connected by an elastic 

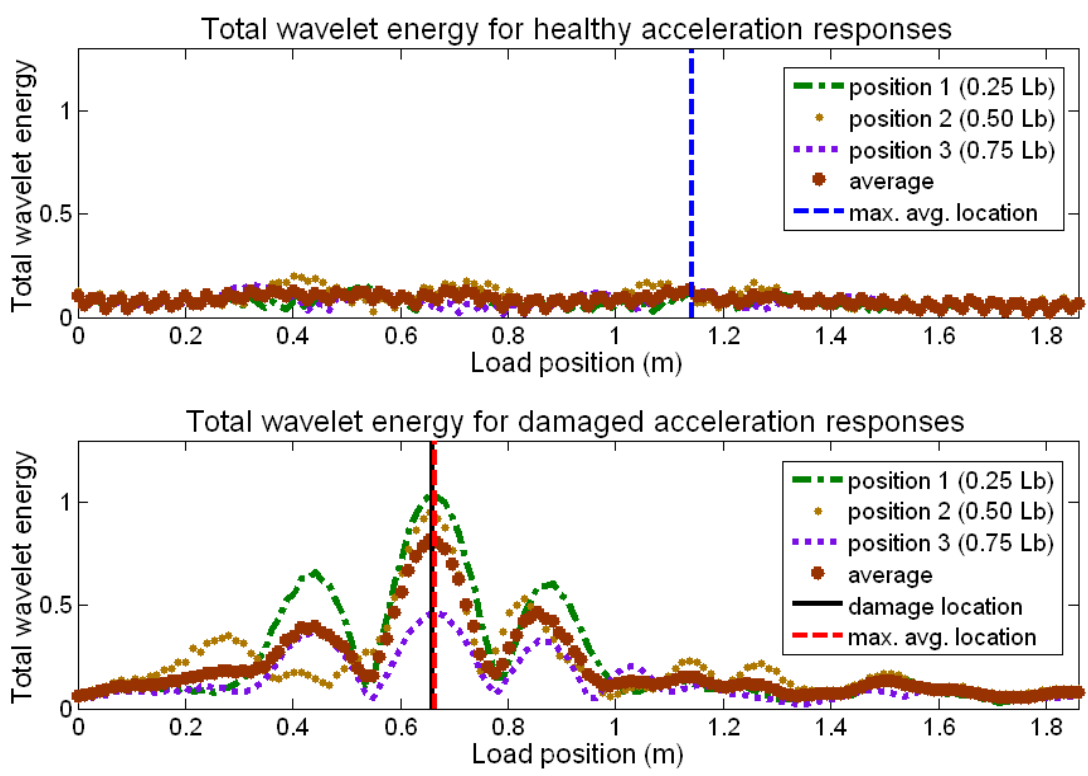

Total average wavelet energy for different cases

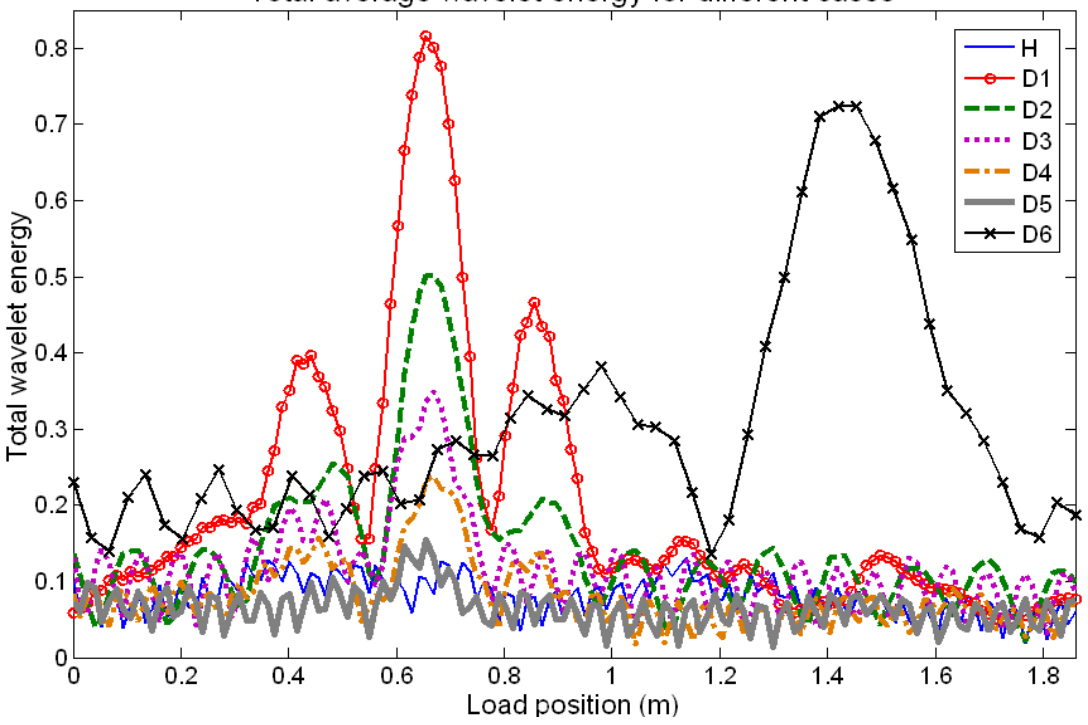

Fig. 4. Total wavelet energy for healthy case $(\mathrm{H})$ and damaged case (D1).
Fig. 5. Total average wavelet energy for all the cases presented in Table 1. aluminum bridge. A ballast was placed on the bridges and the vertical acceleration responses were measured while a wagon passed over the elastic bridge. The mass of the wagon was $0.46 \mathrm{~kg}$ and a mass of $2 \mathrm{~kg}$ was added on the wagon. The dimensions of the elastic bridge were: length $L_{\mathrm{b}}=1.86 \mathrm{~m}$, base $b_{\mathrm{b}}=0.15 \mathrm{~m}$, height $h_{\mathrm{b}}=0.015 \mathrm{~m}$. Damage was represented by attaching a mass of $0.42 \mathrm{~kg}$ on the elastic bridge at $0.66 \mathrm{~m}$ from the left end. The mass simulating damage was equivalent to $2.81 \%$ of the mass of the group ballast-elastic bridge-total moving mass. The wagon was propelled by a system consisting of pulleys, strings, an electric motor, and a DC power supply. The wagon speed was roughly $0.36 \mathrm{~m} . \mathrm{s}^{-1}$. In Figure 6, two pictures of the test-rig are included.

The CWT diagram obtained from the filtered damaged response at $x=0.125 L_{\mathrm{b}}$ is presented in Figure 7 and it is possible to see a significant evidence of damage practically at the exact position where damage was incorporated. This evidence was not observed for the CWT diagram obtained from the noisy damaged response because the noise masked the damage; and therefore, in the interests of brevity, that diagram is not shown.

Finally, the same method described in the numerical part of this article is applied for the experimental results. Thus, the total wavelet energy is calculated for three measuring points of the elastic bridge $\left(0.125 L_{\mathrm{b}}, 0.550 L_{\mathrm{b}}\right.$, and $\left.0.875 L_{\mathrm{b}}\right)$; moreover, the total average wavelet energy is also estimated. The corresponding curves for those wavelet energies are presented in Figure 8 and it can be notified that the maximum peak of energy for each measured point occurs in the neighborhood of the damage location except for the third position, where the energy peak related with the damage is the second one in magnitude. However, if an averaging of the wavelet energies for the different positions is performed, the discrepancies among them can be compensated and, at the end, more 

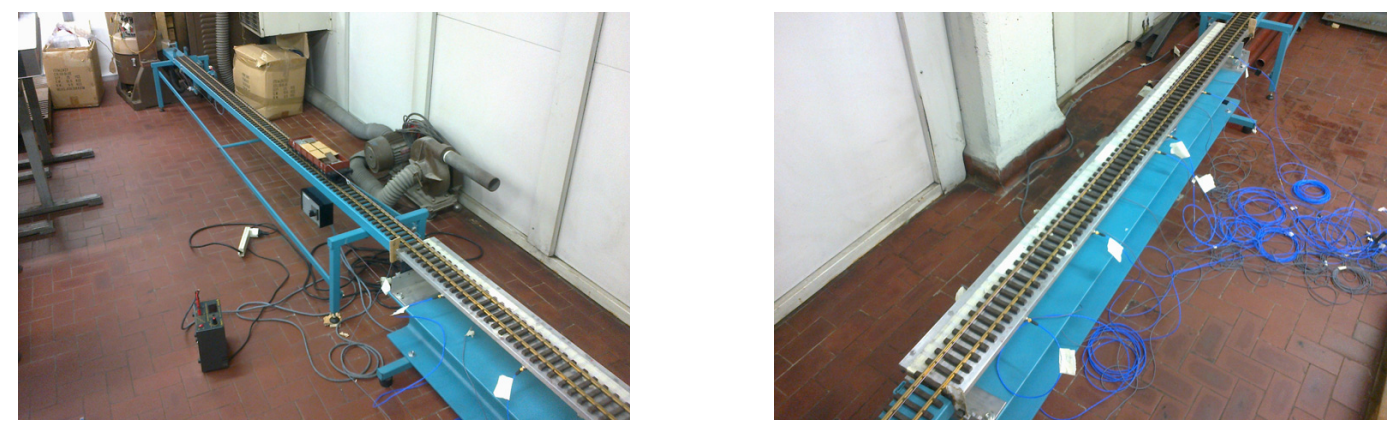

Fig. 6. Pictures of the test-rig showing the wagon before entering to the elastic bridge and the complete instrumented elastic bridge, respectively.
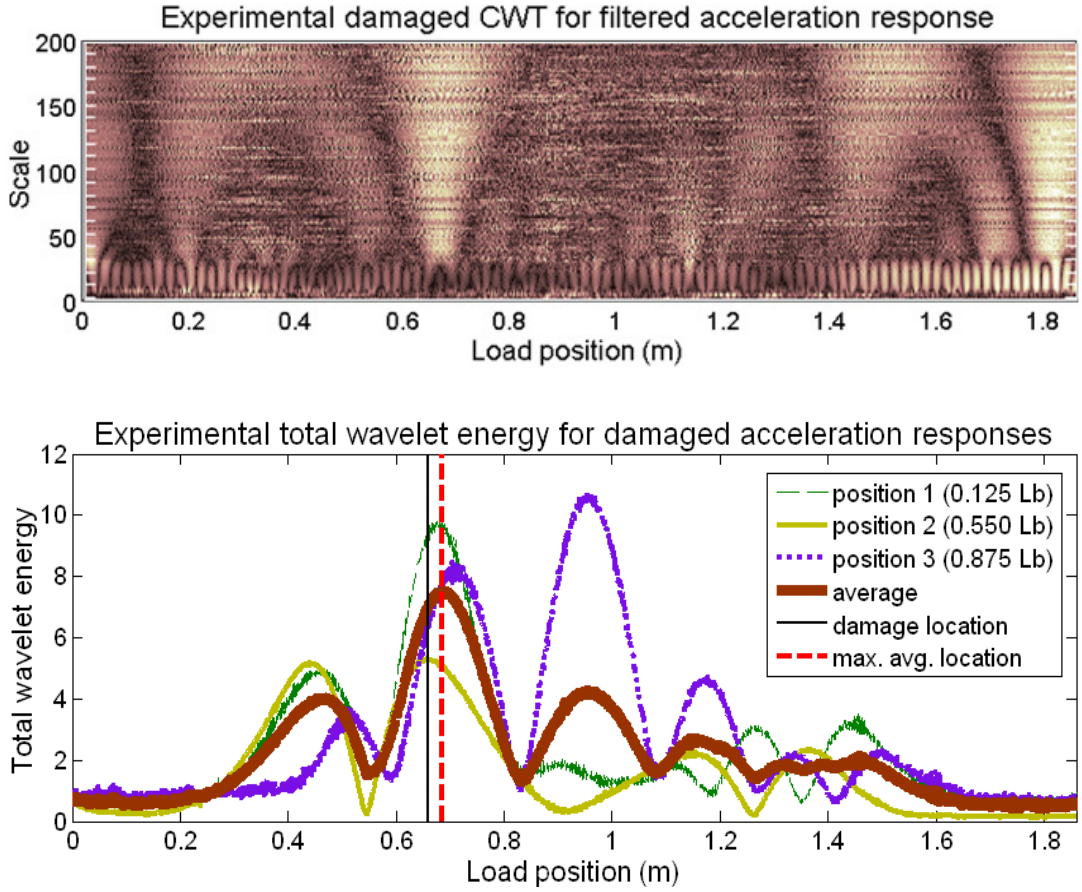

Fig. 7. Experimental filtered CWT diagram for damaged case at $x=0.125 L_{\mathrm{b}}$.

Fig. 8. Experimental total wavelet energy for damaged case. trustful information is obtained. Therefore, if the total average wavelet energy is analyzed, it is possible to see that there is one prominent peak of energy accumulation whose position is very near to the damage position. Consequently, the damage was detected and localized with an error of $3.89 \%$, validating in this way the numerical results.

\section{Conclusions}

In this article we presented an efficient method based on CWT diagrams to detect and localize damage on beam-like structures subjected to moving loads. The method consists in calculating, for many different points along the beam, the wavelet energy from the coefficients along the selected range of scales; thus, a significant peak of wavelet energy accumulation will indicate the presence of damage, whereas its location and intensity could also be estimated according with the position and magnitude of the maximum peak of wavelet energy, respectively. The precision in detecting and localizing damage will increase if the average of a few wavelet energies corresponding to different measuring points is considered. Lastly, it is important that before calculating the wavelet energies, the signals must be filtered and the border effects of the CWT diagrams have to be removed. A good agreement between numerical and experimental results was obtained.

Acknowledgements. The first author thanks to Consejo $\mathrm{Na}$ cional de Ciencia y Tecnología CONACYT-México for the post-doctoral scholarship granted to collaborate with this research.

\section{References}

[1] M. Davey, M.L. Wald, Potential flaw is found in design of fallen bridge, The New York Times, 8, 2007

[2] C.R. Farrar, D.A. Jauregui, Comparative study of damage identification algorithms applied to a bridge: I. Experiment, Smart Mater. Struct. 7 (1998) 704-719 
[3] K.M. Liew, Q. Wang, Application of wavelet theory for crack identification in structures, J. Eng. Mech. 124 (1998) 152-157

[4] X.Q. Zhu, S.S. Law, Wavelet-based crack identification of bridge beam from operational deflection time history, Int. J. Solids Struct. 43 (2006) 2299-2317

[5] C. Surace, R. Ruotolo, Crack detection of a beam using the wavelet transform, Proceedings of the 12th International Modal Analysis Conference, Honolulu, Hawaii, USA, 1994, pp. 1141-1147

[6] S. Quek, Q. Wang, L. Zhang, K. Ang, Sensitivity analysis of crack detection in beams by wavelet technique, Int. J. Mech. Sci. 43 (2001) 2899-2910
[7] E. Douka, S. Loutridis, A. Trochidis, Crack identification in beams using wavelet analysis, Int. J. Solids Struct. 40 (2003) 3557-3569

[8] A. Gentile, A. Messina, On the continuous wavelet transforms applied to discrete vibrational data for detecting open cracks in damaged beams, Int. J. Solids Struct. 40 (2003) 295-315

[9] C.L. Liu, A tutorial of the wavelet transform, 2010

[10] S. Marchesiello, S. Bedaoui, L. Garibaldi, P. Argoul, Time-dependent identification of a bridge-like structure with crossing loads, Mech. Syst. Signal Process. 23 (2009) 2019-2028 